\title{
Modélisation numérique du transport solide en écoulement non permanent Application à la retenue de Belley (Rhône)
}

\author{
Trieu Dong Nguyen, Sung Ho Lee, Philippe Bois \\ Laboratoire d'étude des Transferts en Hydrologie et Environnement * \\ Tormos E., Giuliani Y. \\ Compagnie Nationale du Rhône **
}

\begin{abstract}
L'aménagement de Belley, situé sur le Haut Rhône, fait suite à une succession d'aménagements tels que : Verbois et Chancy - Pougny en Suisse, puis Genissiat, Seyssel et Chautagne du côté français.

Pour évacuer les matériaux solides qui se déposent dans les retenues les plus en amont tous les 3 ans les chasses sont réalisées par les Suisses sur leurs aménagements (les dernières chasses datent de 1993). A l'occasion de ces événements, les ouvrages du côté français gérés par la C.N.R. sont exploités de manière à laisser transiter la plus grande quantité possible de matériaux solides venant de l'amont. Le reste se dépose progressivement dans les retenues aval, suivant les caractéristiques locales du courant et du matériau. Ainsi, s'agissant de l'aménage-
\end{abstract}

ment de Belley, on a mesuré les fonds avant et après les chasses de 1990 , et on a trouvé un volume déposé de l'ordre de $500000 \mathrm{~m}^{3}$.

Il nous est apparu utile de modéliser numériquement l'écoulement et le transport solide dans l'aménagement de Belley, pour prévoir l'évolution du fond, afin d'en déduire les consignes d'exploitation, d'entretien et de transit des crues.

\footnotetext{
* (L.T.H.E.-I.M.G.) (I.N.P.G., U.J.F., C.N.R.S. U.R.A. 1512) B.P. $53 X ; 38041$ Grenoble Cedex.

** (C.N.R., 2 rue A. Bonin, 69316 Lyon Cedex 04).
}

\section{On numerical simulation of unsteady sediment transport} Application on the reservoir of Belley, Rhône

Every three years, the washing operations in the reservoirs of the Upper Rhone let a big amount of sediment flowing downstream. Our simulation aims to describe the mixture flow in the reservoir of Belley. The model encompasses a 16 millions $\mathrm{m}^{3}$ water volume, and a $17 \mathrm{~km}$ long stretch of the Rhone and derivation canal, situated upstream of the hydroelectric plant of Belley. The stretch is divided by a hundred transsects. The incoming flow is laden with very fine sediment (about 10 um mean diameter). The used equations are the St-Venant equations, two sediment mass conservation equations in the waterstream and in the bed-stream, the sediment exchange between waterstream/bedstream equation, and the Van Rijn equations for solid discharge in equilibrium conditions. The rate of solid output volume on input volume determined by the simulation is found in accordance with in-site measures during washing operations. 


\section{Liste des symboles}

$x \quad$ : Abscisse prise le long du bief $(\mathrm{m})$.

$t \quad$ : Temps (s).

Z : Cote de la surface libre $(\mathrm{m})$.

$m \quad$ : Cote du fond $(\mathrm{m})$.

$h \quad$ : Profondeur de la couche de surface $(\mathrm{m})$.

a : Profondeur de la couche de fond $(\mathrm{m})$.

C : Concentration.

$Q \quad$ : Débit liquide $\left(\mathrm{m}^{3} / \mathrm{s}\right)$.

$q$, : Débit liquide latéral par unité de longueur $\left(\mathrm{m}^{2} / \mathrm{s}\right)$.

Q $\quad$ : Débit solide dans la couche de surface (Débit solide en suspension, $\mathrm{m}^{3} / \mathrm{s}$ ).

$Q_{s e} \quad$ : Débit solide à l'équilibre dans la couche de surface $\left(\mathrm{m}^{3} / \mathrm{s}\right)$.

$Q_{b} \quad$ : Débit solide dans la couche de fond (Débit de charriage et saltation, $\mathrm{m}^{3} / \mathrm{s}$ ).

$Q_{b_{c}} \quad$ : Débit solide à l'équilibre dans la couche de fond $\left(\mathrm{m}^{3} / \mathrm{s}\right)$.

$$
R=Q_{s c} / Q_{b c}=Q_{s} / Q_{b} .
$$

: Section mouillée $\left(\mathrm{m}^{2}\right)$.

$A_{b} \quad:$ Aire de la section du fond $\left(\mathrm{m}^{2}\right)$ $u \quad:$ Vitesse $(u=Q / A)(\mathrm{m} / \mathrm{s})$.

$u_{i r} \quad:$ Vitesse moyenne critique, d'après Shields $(\mathrm{m} / \mathrm{s})$.

u. : Vitesse de cisaillement $(\mathrm{m} / \mathrm{s})$

$K_{c} \quad$ : Coefficient des pertes de charge singulières.

$S_{f} \quad$ : Pente de frottement.

$n \quad$ : Coefficient de Manning-Strickler.

ks : Rugosité équivalente du fond $(\mathrm{m})$.

$C^{\prime} \quad:$ Nombre de Chézy relatif au grain, $\left(\mathrm{m}^{1 / 2} / \mathrm{s}\right)$.

$R_{h} \quad:$ Rayon hydraulique $(\mathrm{m})$.

$\kappa \quad$ : Constante de Prandtl (0.4).

$p \quad$ : Porosité du sédiment $(p=0.4)$.

$s \quad:$ Densité du sédiment $(s=2.7)$.

$L^{*} \quad$ : Longueur caractéristique de l'échange surface/fond $(\mathrm{m})$.

$S \quad$ : Débit d'échange surface/fond par unité de longueur $\left(\mathrm{m}^{2} / \mathrm{s}\right)$

$d_{90 f} \quad$ : Diamètre à $90 \%$ du matériau du fond, déterminé par dragages $(\mathrm{m})$.

$d_{16}, d_{50}$, etc : Diamètre du sédiment suivant différents \% de masse $(\mathrm{m})$.

$D^{*} \quad$ : Diamètre adimensionnalisé du sédiment $\left(D^{*}=d\left(g \cdot(s-1) / v^{2}\right)^{1 / 3}\right)$.

$\varepsilon_{s} \quad:$ Coefficient de diffusion longitudinale $\left(\mathrm{m}^{2} / \mathrm{s}\right)$.

\section{Description de l'aménagement}

L'aménagement de Belley intéresse une longueur du Rhône de $22 \mathrm{~km}$ environ. Il se compose, de l'amont vers l'aval (fig. l).

1. D'une retenue, de longueur $4,5 \mathrm{~km}$ dans le lit naturel du Rhône et à l'extrémité aval de laquelle est implanté un barrage équipé de 4 vannes-segments. Elle est bordée :

a) en rive droite, d'une digue insubmersible de $4,5 \mathrm{~km}$ de long:

b) en rive gauche, d'une berge submersible de $2 \mathrm{~km}$ suivi d'une digue insubmersible de $2,5 \mathrm{~km}$.

2. D'un canal d'amenée à l'usine hydroélectrique de Brens. De $13,5 \mathrm{~km}$ de longueur, ce canal emprunte la

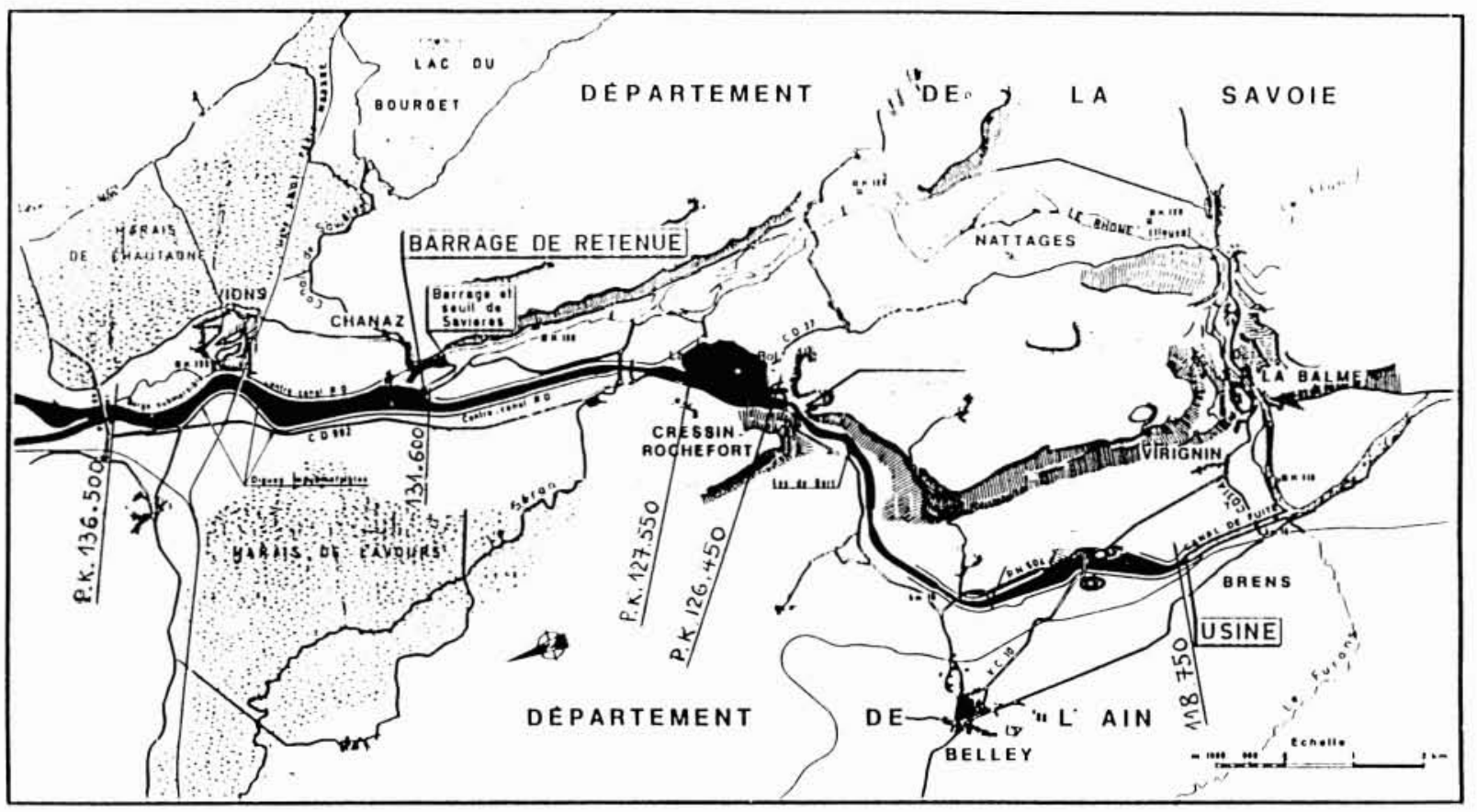

1. Plan d'ensemble de l'aminagement. 


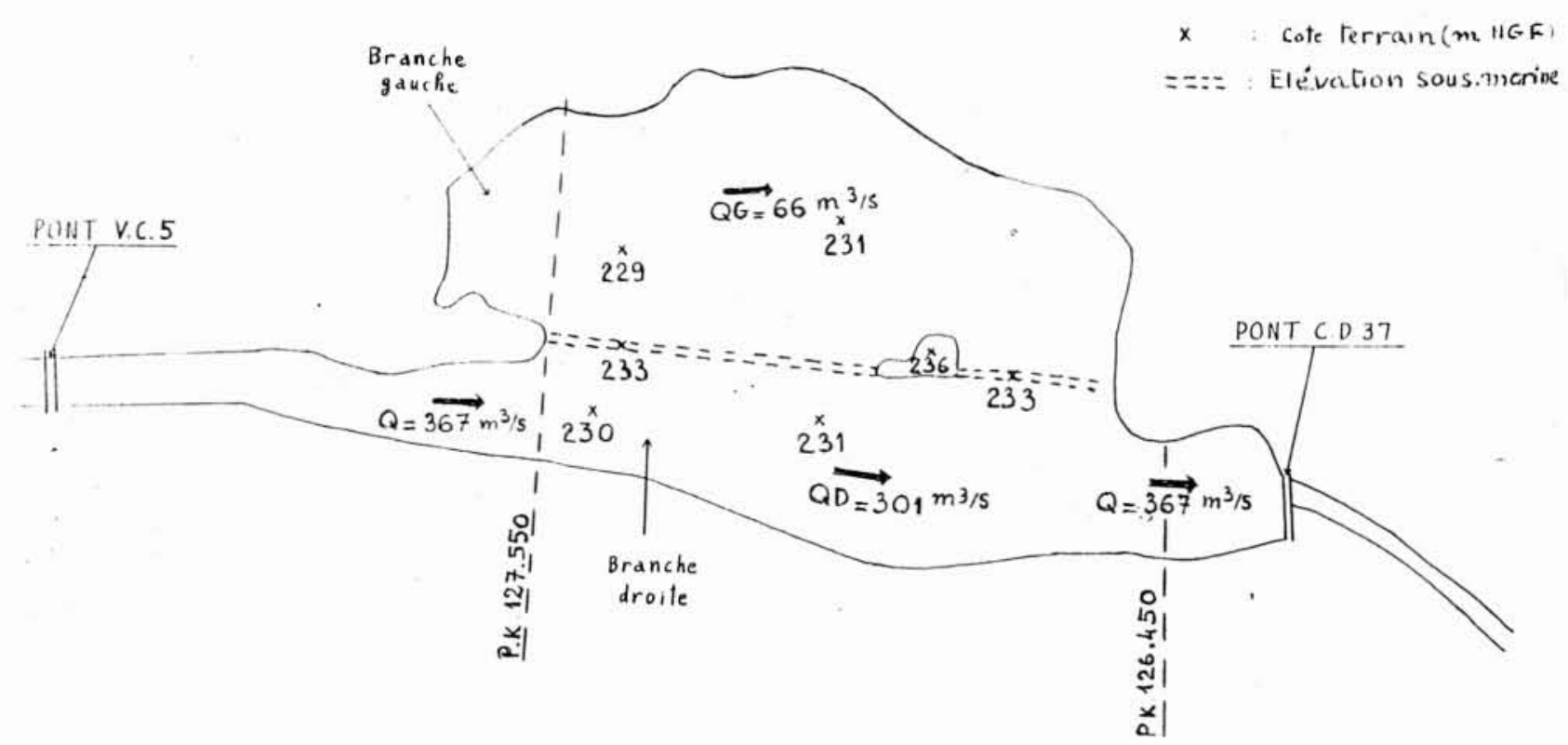

2. Marais de Cressin.

dépression constituée par le marais de Cressin et le lac de Bart.

Dans le marais du Cressin, l'écoulement est partagé en deux branches par une surélévation longitudinale du fond qui culmine vers le milieu du lac par un îlot (fig. 2). Sur les deux branches en rive droite (RD) et en rive gauche (RG), nous avons des vitesses assez différentes (donc des capacités de transport solide différentes), avec la majeure partie du débit passant dans la branche en RD. Par la suite, nous devons tenir compte de cette particularité dans la modélisation de l'hydraulique du marais.

\section{Le modèle de transport solide (fig. 3)}

Pendant les chasses, les matériaux mobilisés sont très fins, avec un diamètre inférieur à $100 \mu \mathrm{m}$, et un $d_{50}$ de l'ordre de $10 \mu \mathrm{m}$ (chasses de 93) (voir courbes en $S$, fig. 4).

C'est pourquoi dans notre modélisation nous devons tenir compte du transport de fond et du transport en suspension, des phénomènes de soulèvement, de chute surtout à l'interface entre la zone de transport de fond et la zone de transport par suspension. Le transport par suspension occupe la majeure partie de la masse d'eau et celui par le fond occupe la partie restante. Le terme d'échange à l'interface de la couche de surface/couche de fond est noté $S$. Cette schématisation a été notamment

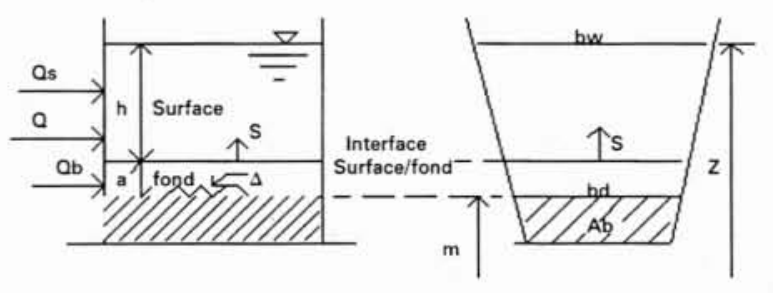

3. Schéma du transport solide.

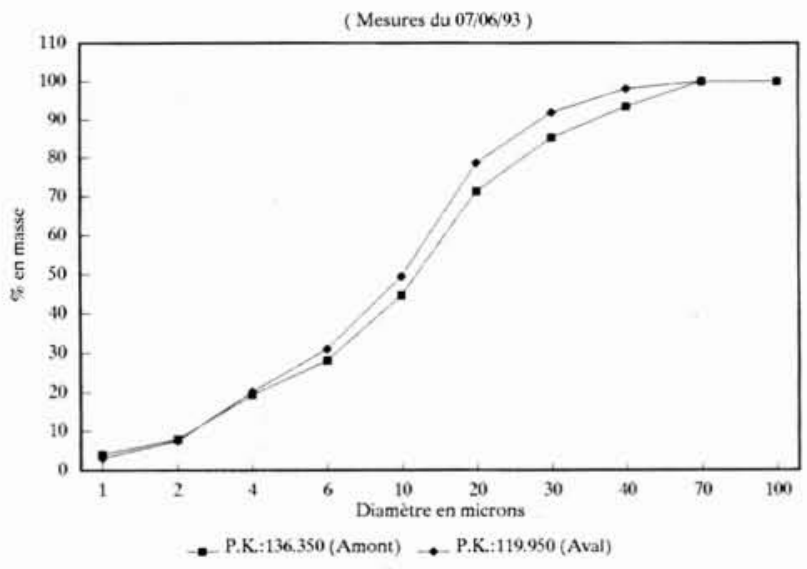

4. Granulométrie du sédiment en suspension, mesurée le 07/06/93.

utilisée par AmANINI et SILvio [4]. Les inconnues de notre modèle sont la cote de la surface libre $Z(x, t)$, le débit liquide $Q(x, t)$, la cote du fond mobile $m(x, t)$ et la concentration de matière en suspension $C(x, t)$.

Nous utilisons, pour calculer les débits solides de fond et en suspension, les formules de VAN RIJN [1], [2], [3]. Pour comparer le débit solide total (de fond et en suspension) ainsi calculé avec le débit solide total donné par d'autres auteurs, nous avons employé les formules d'ACKERS et White [6], et celles d'ENGELUND et HANSEN [7].

\section{Système d'équations du modèle}

Le système d'équations considéré se compose des équations de St. Venant, des équations donnant la rugosité du 


\section{MODÉLISATION EN ÉCOULEMENT NON PERMANENT}

lit, des équations de conservation de la masse du matériau dans la couche de transport par suspension et dans la couche de transport de fond, des équations du débit solide en suspension et en charriage.

\subsection{Equations de Saint Venant}

Equation de continuité

$$
\frac{\partial Q}{\partial x}+\frac{\partial A}{\partial t}=-q_{r} .
$$

Le débit latéral $q_{r}$ est positif lorsque l'eau sort du modèle par les déversoirs du barrage ou, en cas de crue, par déversement au-dessus de la digue submersible.

Equation du mouvement

$$
\frac{\partial Q}{\partial t}+\frac{\partial}{\partial x}\left(\frac{Q^{2}}{A}\right)+\frac{\partial Z}{\partial x}+g A S_{f}+\frac{K_{e} A}{2} \frac{\left|\partial u^{2}\right|}{\partial x}=0 .
$$

La pente $S_{f}$ des pertes de charge régulières est donnée par :

$$
S_{f}=\frac{n^{2} Q|Q|}{A^{2} R_{h}^{4 / 3}} .
$$

Le coefficient $n$ est déterminé de la manière suivante :

$$
n=n_{\text {base }}+n_{\text {sup }} \text {. }
$$

La valeur de $n_{\text {base }}$ dépend de la nature du fond: dimension du matériau tapissant le fond et présence des rides ou dunes sur ce fond. Le diamètre du matériau, ainsi que la hauteur $\Delta$ et la longueur d'onde $\Lambda$ des ondulations variant suivant les caractéristiques de l'écoulement et du transport solide, le coefficient $n_{\text {base }}$ est initialement estimé suivant les tables du S.C.S. [5], puis redéterminé à chaque pas de calcul de la manière suivante.

On calcule la rugosité équivalente $k s$ du fond par

$k s=k s$ grain $+k s$ ride avec

$k s$ grain $=3^{*} d_{90 f}$ et $k s$ ride $\cong \Delta$ (hauteur de la ride)

(Le $d_{90 f}$ est déterminé suivant les prélèvements du fond, effectués en septembre 91. Par exemple dans le Marais de Cressin, $d_{90 f}$ est de l'ordre de $140 \mu \mathrm{m}$ ).

A partir de cette rugosité $k s$, on calcule le nombre de Chézy relatif au grain $C^{\prime}$ :

$$
C^{\prime}=\frac{\sqrt{g}}{\kappa} \operatorname{Ln}\left(12 \frac{R_{h}}{k_{s}}\right)
$$

et on détermine $n_{\text {base }}$ à partir de :

$$
C^{\prime}=\frac{1}{n_{\text {base }}} R_{h}^{1 / 6} .
$$

Ce coefficient caractérise ainsi l'interaction entre l'écoulement biphasique liquide-solide et l'état de la surface du fond. Quant au paramètre $n_{\text {sup }}$ caractérisant les singularités (ouverture ou rétrécissement brusque, divagation du thalweg d'une berge à une autre), il est gardé constant dans notre calcul.

Voici un tableau donnant les valeurs de $n$ de la limite amont jusqu'au Marais de Cressin :

Tableau I. - Exemples des valeurs de $\boldsymbol{n}$ sur une partie du modèle.

\begin{tabular}{|c|c|l|c|}
\hline Du P.K. amont & au P.K. aval & \multicolumn{1}{|c|}{ Description du tronçon } & $n$ \\
\hline 136500 & 133400 & Amont du modèle. Partie Rhône nature avec divagation du thalweg & 0,033 \\
133400 & 131600 & Amont du modèle. Partie Rhône nature sans divagation du thalweg & 0,028 \\
131600 & 129650 & Canal artificiel, rectiligne, fond plat & 0,025 \\
129650 & 128650 & Canal artificiel, rectiligne, fond avec fosse (fosse du Séran) & 0,028 \\
128150 & 127550 & Entrée du Marais de Cressin & 0,028 \\
127550 & 126600 & Marais de Cressin, branches RD et RG & 0,025 \\
\hline
\end{tabular}

\subsection{Equations de conservation du matériau solide}

3.2.1. Conservation dans la couche de surface (waterstream)

$$
\frac{\partial C A}{\partial t}+\frac{\partial Q_{s}}{\partial x}=S-q_{r} C+\frac{\partial}{\partial x}\left(A \varepsilon_{s} \frac{\partial C}{\partial x}\right) .
$$

\subsubsection{Conservation dans la couche de fond}

$$
(1-p) \frac{\partial A_{b}}{\partial t}+\frac{\partial Q_{b}}{\partial x}=-S .
$$

Le terme d'échange $S$ est donné par ARMANINI et DI SILvio [4] par

$$
S=\frac{1}{L^{*}}\left(Q_{s e}-Q_{s}\right)
$$

Ainsi quand le débit en suspension actuel $Q_{s}$ est plus grand que le débit d'équilibre $Q_{s e}(S<0)$, c'est la couche de surface qui alimente le fond en matériau solide. VAN RIJN [3] a donné les formules suivantes pour les débits solides à l'équilibre.

Pour la couche de fond :

$$
\begin{aligned}
& Q_{b e}= \\
& \quad=0,005\left[\frac{u-u_{c r}}{\left\{(s-1) g d_{50}\right\}^{0.5}}\right]^{2,4}\left(\frac{d_{50}}{h}\right)^{1.2} u h b_{d} .
\end{aligned}
$$

Pour la couche de surface :

$$
\begin{aligned}
Q_{s e}=0,012\left[\frac{u-u_{c r}}{\left\{(s-1) g d_{50}\right\}^{0,5}}\right]^{2,4}\left(\frac{d_{50}}{h}\right) \times \\
\times\left(D^{*}\right)^{-0.6} u h b_{d} .
\end{aligned}
$$


Ici le rapport $\left.R=Q_{s}\right\rfloor Q_{b e}$ ne dépend que du grain et de la profondeur. Nous supposons que même en déséquilibre, le rapport des débits de surface/fond reste le même, c.-à-d. :

$$
R=\frac{Q_{s}}{Q_{b}}=\frac{Q_{s e}}{Q_{b e}} .
$$

Le terme de diffusion longitudinale dans (7) est considéré comme un terme correctif. Il est calculé explicitement avec les valeurs des paramètres définies à l'instant précédent, et avec [8] :

$$
\varepsilon_{s}=10 \cdot u_{*} \cdot h \text {. }
$$

Pour résoudre notre système d'équations, nous avons appliqué le schéma implicite de Preissmann sur les différences finies, et nous avons étendu la méthode du double balayage au cas d'une divergence-confluence.

\section{Le modèle hydraulique}

Le bief modélisé a une longueur de $17,750 \mathrm{~km}$. Le volume d'eau pris en compte est de l'ordre de $16,852 \mathrm{hm}^{3}$ (lorsque le débit est de $700 \mathrm{~m}^{3} / \mathrm{s}$ et que la cote NGF au barrage est de $234,80 \mathrm{~m}$ ). L'écoulement peut être considéré comme unidirectionnel (unifilaire) presque partout, sauf dans le Marais de Cressin, où une surélévation longitudinale du fond culminant par un îlot au milieu du marais nous oblige à y schématiser l'écoulement sous forme d'une maille (fig. 5). Sur cette figure,

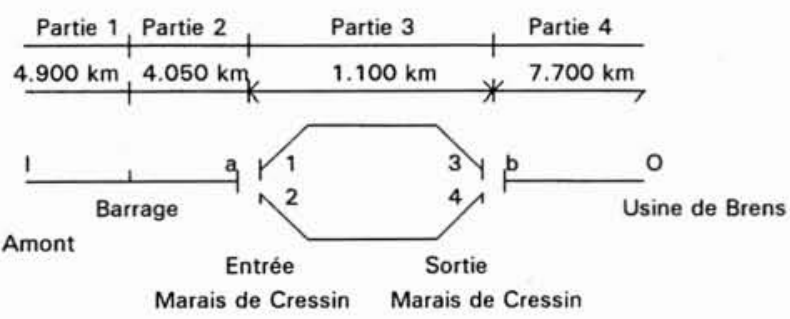

Qi donné

Ci donné

Zo donné Mo donné

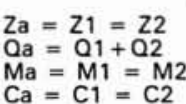

$\mathrm{Ma}=\mathrm{M1}=\mathrm{M}$

$\mathrm{Ca}=\mathrm{C} 1=\mathrm{C} 2$

$\mathrm{Zb}=\mathrm{Z3}=\mathrm{Z4}$

$\mathrm{ab}=\mathrm{Q} 3+\mathrm{Q} 4$

$\mathrm{Si} M 4>\mathrm{M} 3$ alors $\mathrm{Mb}=\mathrm{M} 4$

$\mathrm{Si} \mathrm{M} 3>\mathrm{M} 4$ alors $\mathrm{Mb}=\mathrm{M} 3$

$\mathrm{Cb} . \mathrm{Qb}=\mathrm{C} 3 . \mathrm{O} 3+\mathrm{C} 4.04$

5. Schéma du modèle.

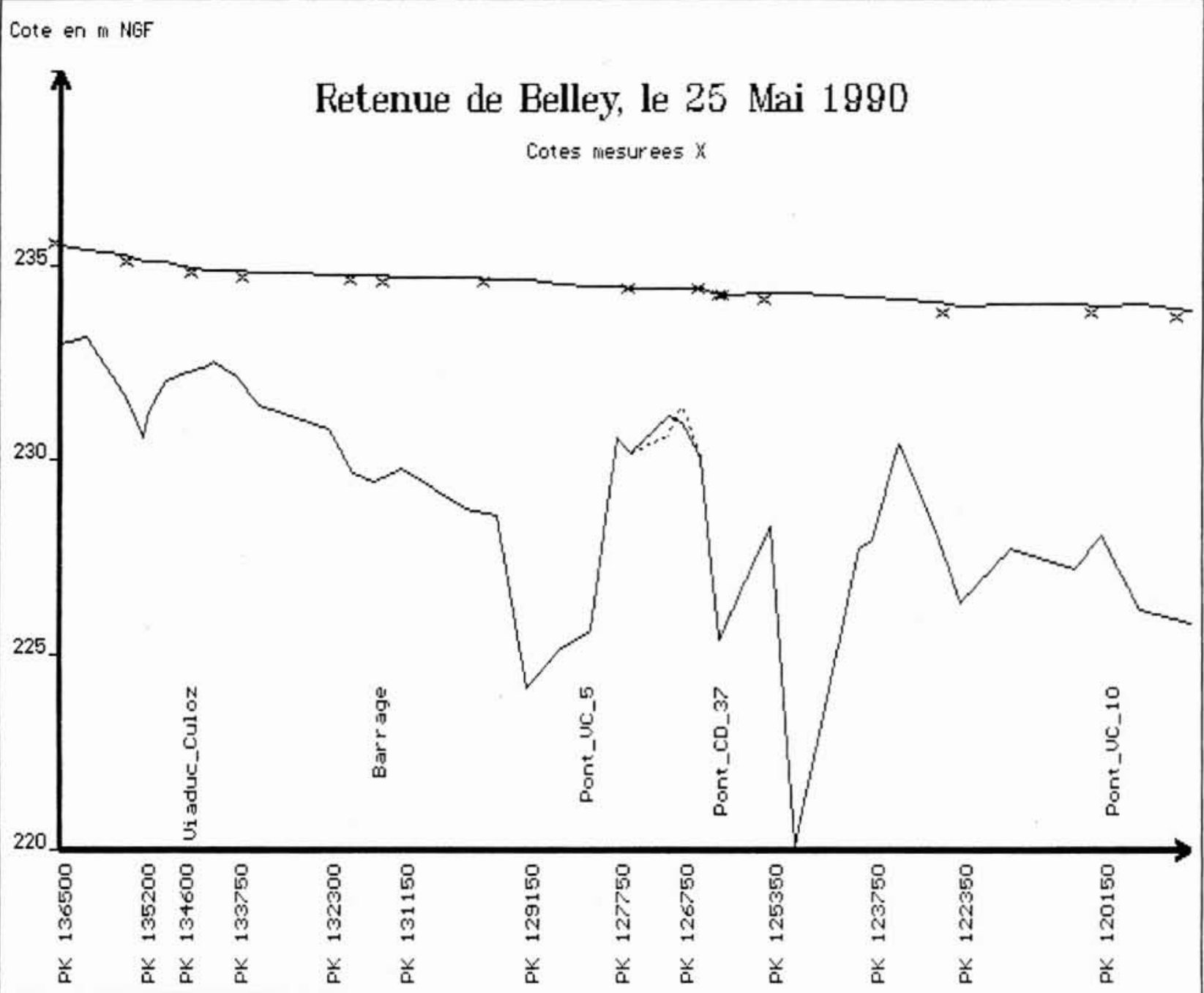

6. Ligne d'eau calculée et mesurée, ligne de fond mesurée. 
nous donnons aussi les conditions aux limites et aux points de défluence et d'affluence.

\section{Résultats de la modélisation}

Nous avons d'abord vérifié le modèle hydraulique avec fond fixe sur différentes lignes d'eau mesurées par la C.N.R. La comparaison entre cote d'eau mesurée et cote d'eau calculée donne une erreur maximum de l'ordre de $10 \mathrm{~cm}$, ce qui est acceptable étant donné que la mesure d'une ligne d'eau sur les $17 \mathrm{~km}$ de longueur de la retenue nécessite un temps d'au moins une heure, au cours duquel les cotes peuvent varier dans cet intervalle.

Ensuite nous avons pris les données hydrauliques du 25 mai 1990 (débit entrant $715 \mathrm{~m}^{3} / \mathrm{s}$, débit barrage $28 \mathrm{~m}^{3} / \mathrm{s}$, débit usine $667 \mathrm{~m}^{3} / \mathrm{s}$, cote à l'usine $233,90 \mathrm{~m} \mathrm{NGF}$ ), journée où on avait fait des mesures de cote de surface libre et dont le débit est sensiblement celui observé pendant les chasses. Les cotes calculées de la surface libre et celles du fond sont données dans la figure 6 . Les débits sont donnés figure 7.
Sur la figure 4, nous avons les granulométries mesurées au Belley pendant les dernières chasses de juin 93 , en deux sections situées en amont et en aval du modèle. Pour la section située en amont, et à mi-profondeur, les diamètres $d_{16}, d_{50}, d_{70}$ et $d_{84}$ sont respectivement égaux à $3,11,20$ et $30 \mu \mathrm{m}$.

Nous supposons alors une entrée de sédiment de diamètre uniforme $d=0,11 \mu \mathrm{m}$. Nous avons pris une variation de la concentration avec temps analogue à celle mesurée à Vions, pendant les chasses de 90 (voir courbe 1 de la figure 8 ): la durée de cette bouffée de sédiment est de deux jours, avec une pointe de concentration de $8 \mathrm{~g} / \mathrm{l}$ en fin de la première journée. La figure 8 donne les concentrations calculées à différentes sections.

Sur la figure 9, nous avons le volume cumulé en différentes sections, en milliers de $\mathrm{m}^{3}$. Pendant ces deux jours, le volume solide entrant dans le modèle est d'environ $224500 \mathrm{~m}^{3}$. Le volume qui en est sorti est de $164900 \mathrm{~m}^{3}$. Le volume déversé à travers le barrage étant de $8400 \mathrm{~m}^{3}$, nous avons un volume de $51200 \mathrm{~m}^{3}$ qui s'est déposé dans la retenue (soit $23 \%$ du volume à l'entrée).

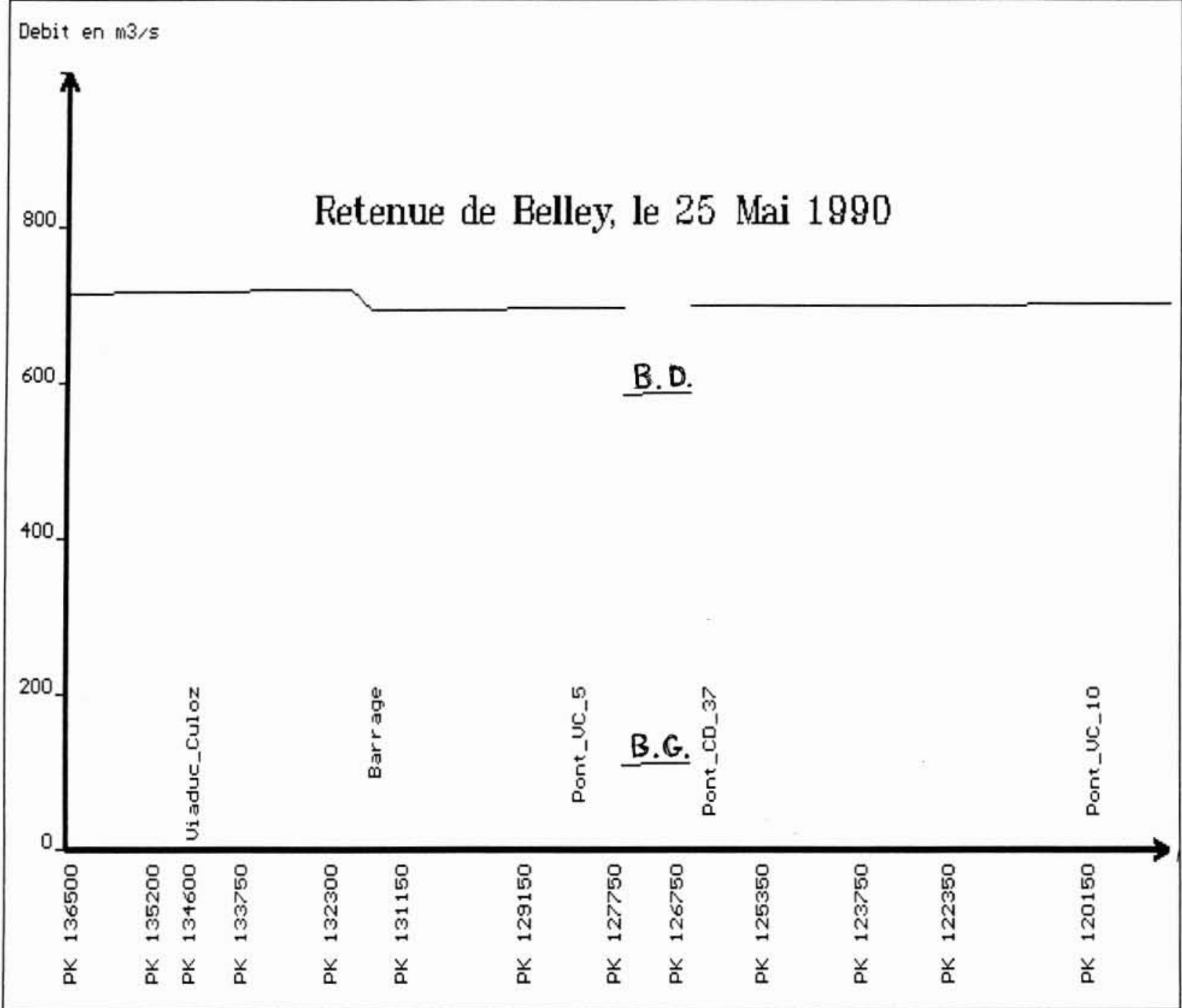

7. Débit le long de la retenue. 